\title{
Study of Series-Parallel and Power-Split DHT for Hybrid Powertrains
}

\author{
Haijun Chen ${ }^{1} \cdot$ Lin Li $^{1}$ • Ferit Küçükay ${ }^{1}$
}

Received: 7 January 2020 / Accepted: 13 November 2020 / Published online: 29 January 2021

(c) The Author(s) 2021

\begin{abstract}
Dedicated hybrid transmission (DHT) is the developing trend of hybrid transmissions. This paper studies different types of DHTs regarding the structure, function, and performance. Firstly, the structure and function of different well-known DHTs in the market are discussed and analyzed. Through the analysis, the reasonability and necessity of the different modes and the electric motor power requirements that lie behind different DHTs are derived. Secondly, the dynamics of different DHTs are further compared with the driving areas in different modes under different driving conditions. Then, the basic or minimum dynamic requirements are defined and taken into consideration before the economic comparison. To ensure the effectiveness of the comparison, the optimization of the configurations for each kind of DHT is carried out with the modular simulation model. The economic comparison is conducted under the premise of excluding the influences of the configurations in the results. Finally, the sensitivity and influence of different engine characteristics for different DHTs are studied to find out the sensibilities of the two kinds of DHTs with engine technology. Through these studies, the essential differences and features of different DHTs can be derived to help better understand the decision in choosing the technical route for the original equipment manufacturers.
\end{abstract}

Keywords Dedicated hybrid transmission · Series-parallel DHT $\cdot$ Power-split DHT $\cdot$ Mechanical complexity $\cdot$ Electrical complexity

$\begin{array}{ll}\text { Abbreviations } \\ \text { AT } & \text { Automatic transmission } \\ \text { AMT } & \text { Automatic manual transmission } \\ \text { DCT } & \text { Dual clutch transmission } \\ \text { DHT } & \text { Dedicated hybrid transmission } \\ \text { eCVT } & \text { Electronic continuously variable transmission } \\ \text { EM } & \text { Electric motor } \\ \text { EV } & \text { Electric vehicle } \\ \text { HCU } & \text { Hybrid control unit } \\ \text { HEV } & \text { Hybrid electric vehicle } \\ \text { ICE } & \text { Internal combustion engine } \\ \text { MT } & \text { Manual transmission } \\ \text { OEM } & \text { Original equipment manufacturer } \\ \text { OWC } & \text { One-way clutch } \\ \text { PHEV } & \text { Plug-in hybrid electric vehicle }\end{array}$

Haijun Chen

haijun.chen@tu-braunschweig.de

1 Institute of Automotive Engineering (IfF), Technische Universität Carolo-Wilhelmina zu Braunschweig, Braunschweig, Germany

\section{Introduction}

There are two powertrain solutions for plug-in hybrid electric vehicle (PHEV)/hybrid electric vehicle (HEV): add-on hybrid and dedicated hybrid transmission (DHT). Much research has already agreed that DHT represents the development trend of hybrid transmissions, rather than the add-on hybrid [1,2]. Generally speaking, according to the structure and function, all the DHTs existing in the market can be further divided into two categories: series-parallel DHT and power-split DHT. Concerning power-split DHT, it can be further divided into one-mode DHT and two-mode DHT according to the number of the power-split modes. For longterm development, the DHT solution has a greater advantage due to low cost and better performance in comparison with an add-on solution, like P0 and P2 [3].

But what are the features of different DHTs in the structure and function? Especially for the well-known DHTs, what advantages enable their superiority? Besides, DHT needs to be configured in the powertrain. Even with the same DHT layout, the performance can be largely different when matched with different parameters and power sources in the same vehicle [4]. The influence of configurations on 
the DHT performance should be excluded from the comparison, so that the DHTs could be compared under the optimal configurations.

Thus, the comparison between series-parallel DHT and power-split DHT is conducted herein. To study these two kinds of DHTs, the existing representative DHTs in the market are taken as examples. The analysis will be carried out from the perspective of structure, function, and performance regarding dynamics and economics. This paper is organized as follows: The comparison of series-parallel and power-split DHT in structure is described in Sect. 2. Then the dynamic analysis for the different types of DHTs in different driving situations is introduced in Sect. 3. Sect. 4 gives a description of the simulation model and the corresponding optimal control strategy for the simulation. Finally, the results of the economic analysis are presented in Sect. 5.

\section{Structure of Series-Parallel DHT and Power-Split DHT}

To compare different types of DHTs, the following DHT products in the market are chosen as examples:

1. Series-parallel DHT: Honda Accord i-MMD;

2. One-mode DHT: Toyota Prius THS-IV;

3. Dual-mode DHT: GM Voltec II.

These typical DHTs have already been brought into the market for a long time. Even the division of DHTs is because of the existence of these typical ones. They can be counted as the best DHTs in their catalogs. To illustrate the essential differences, the layouts and functions of different DHTs are first demonstrated.

\subsection{Series-Parallel DHT}

Series-parallel DHT is well known for its simple structure and working principle. The original equipment manufacturers (OEMs) with the transmission technology of automatic manual transmission (AMT) or dual clutch transmission (DCT) can easily transform their hybrid products to this type. Although three generations of Honda Accord i-MMD have been developed, the structure and function of the i-MMD system remain the same. The main working principle of series-parallel DHT is to improve engine fuel consumption through series mode in low vehicle speed and through parallel or engine-solo mode in high vehicle speed, because the economic performance of the overall system in high vehicle speed is much better for directing engine driving without extra power lost from power transition. The OEMs with mature engine technology can choose this technical solution as well. The layout of the Honda Accord i-MMD system is displayed in Fig. 1.

The mechanical structure of Honda Accord i-MMD system is extremely simple. There exists only one clutch to separate the engine and wheel. The mechanical complexity of this layout is very low. But the maximal power of these EMs stays both above $100 \mathrm{~kW}$ to fully release the functional potential of this layout. Especially, in electric vehicle (EV) or series mode, only electric motor 2 (EM2) can drive the vehicle alone without the aid of electric motor 1 (EM1).

The realized modes of the series-parallel DHT are demonstrated in Fig. 2, in which:

1. At low speed or battery SOC high: EV mode;

2. At middle speed or battery SOC low: series mode, the engine is used to charge the generator EM1, and EM 2 will drive the vehicle by itself;

3. At high speed: due to the high efficiency of direct driving by the engine, the engine-solo or parallel mode is adopted.

After analyzing different series-parallel DHTs, such as VW Twin Drive and GAC G-MC system, it is found that all of those DHTs possess the same principle in function and the same basic layout in structure. It means that for all layouts of this type of DHT, there is always one gear ratio or direct connection between the engine and generator. Between the engine and wheel, there always exists one or two gear engagements. There is a gear ratio between the driving motor and the wheel as well. Only the number and position of the gears vary. Besides, after the studies, it is near impossible to find out any other series-parallel DHT that are simpler than Honda Accord i-MMD, VW Twin Drive, and GAC G-MC.

\subsection{Power-split DHT}

Structurally, the power-split DHT is built with planetary gear sets. According to the ways of power-split, it can be further divided into input-, output-, and compound-power-split.

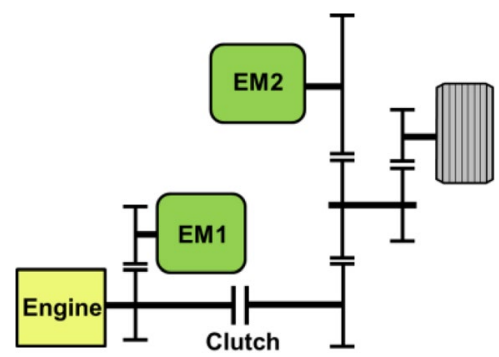

Fig. 1 The layout of Honda Accord i-MMD system 
Fig. 2 The working modes and power flows of Honda Accord i-MMD system

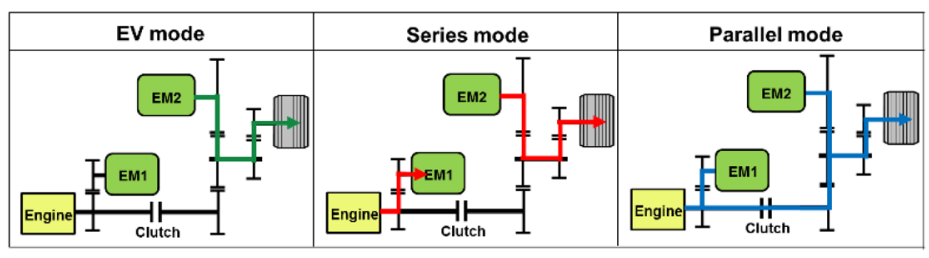

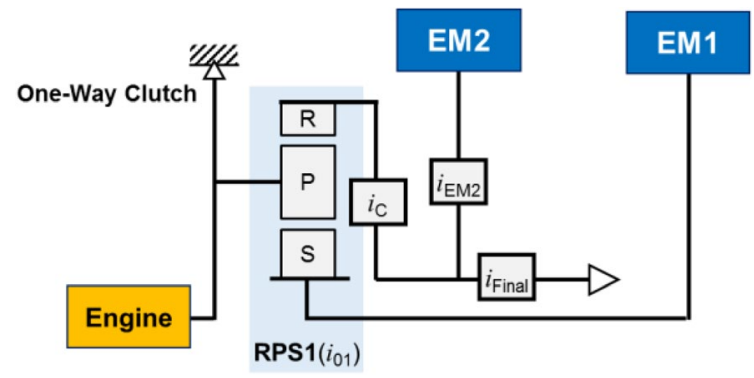

Fig. 4 The simplified model of THS I-IV

Table 1 The realized modes of THS-IV and shifting element

\begin{tabular}{ll}
\hline Mode & One-way clutch \\
\hline 1 EM-EV & \\
2 EM-EV & \\
Input-eCVT (Input-power-split) & \\
\hline
\end{tabular}

when two electric motors jointly drive the vehicle, which increases the maximum vehicle speed and dynamics in $\mathrm{EV}$ driving mode. The one-way clutch doesn't need the actuator and clutch control, but the EM1 needs to be precisely controlled to balance the engine torque when the engine is started. In all the THS generations, the simplified model always remains the same, as seen in Fig. 4, in which S, P, and $\mathrm{R}$ represent the sun gear, planetary gear, and ring gear, respectively. The gear ratio $i_{\mathrm{EM} 2}$ between EM2 and output and the gear ratio $i_{\mathrm{C}}$ between the planetary gear set and output are realized in different mechanical solutions, like planetary gear set with a fixed component or parallel shaft gears.

Input-power-split, auch input-eCVT is the only hybrid mode in THS-IV, and it enables the driving of the vehicle from low to high speed with moderate fuel consumption in one hybrid mode. Under the extremely high vehicle speed, the power circulation will appear, such high speed is rarely used in the normal driving conditions in China but 


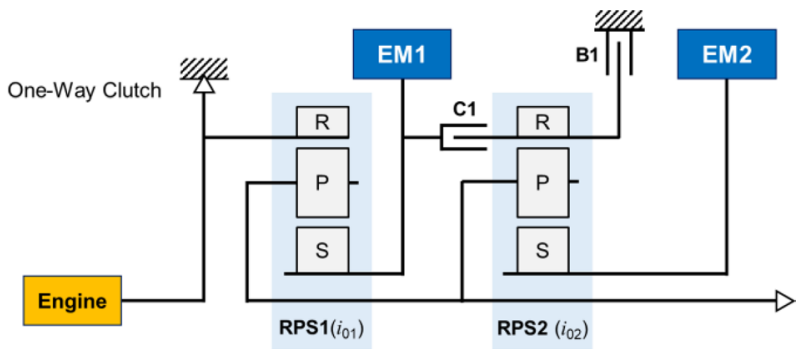

Fig. 5 The layout of Voltec II

Table 2 The realized modes of Voltec II and means the closed shifting element

\begin{tabular}{llll}
\hline Mode & $\mathrm{Bl}$ & $\mathrm{Cl}$ & One-way clutch \\
\hline 1 EM-EV & $\bullet$ & & \\
$2 \mathrm{EM}-\mathrm{EV}$ & - & & \\
$\begin{array}{l}\text { Input-eCVT (Input- } \\
\text { power-split) }\end{array}$ & - & & \\
$\begin{array}{l}\text { Parallel } \\
\begin{array}{l}\text { Compound-eCVT (Com- } \\
\text { pound-power-split) }\end{array}\end{array}$ & - & - & \\
\hline
\end{tabular}

necessary in the USA and European markets. The realized driving modes of THS-IV are displayed in Table 1.

\subsubsection{Dual-mode DHT: Voltec II}

The second example of power-split DHT is Voltec II as shown in Fig. 5. Its structure is more complicated than THSIV. Compared with THS-IV, one more planetary set and two more shifting elements (B1, C1) are involved in this coaxial layout. In addition to the functions or driving modes realized by THS-IV, two more driving modes, namely compoundpower-split (compound-eCVT) and parallel mode [9], are realized in the Voltec II to improve efficiency under high vehicle speed. General Motor, for the first time, combines the input- and compound-power-split in one DHT, which benefits the economic performance and the reduction of the
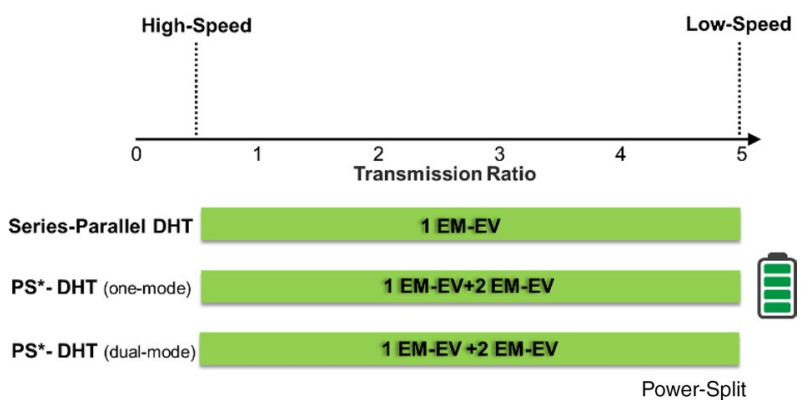

Fig. 6 The comparison of mode distribution in CD mode power demand for two electric motors [10]. The realized modes of Voltec II are displayed in Table 2.

\subsection{Analysis of the DHTs in Function}

The modes and functions of different DHTs are compared in this section to find out how the modes in different DHTs are combined and how these modes are applied in different driving situations. For PHEVs, the driving situations are classified into two kinds: charge-depleting (CD) mode and charge-sustaining (CS) mode, according to the energy management in the hybrid control unit (HCU).

1. CD mode: in this situation, purely electric driving is possible to drive the vehicle.

2. CS mode: the battery cannot output any energy and purely electric driving is impossible, but two electric motors can be involved in the driving with the power generated from the engine to maintain the minimum allowed SOC level.

In the $\mathrm{CD}$ mode, the distribution of modes and function in different DHTs are shown in Fig. 6. The $x$-axis is the transmission ratio varying from low speed to high speed from the right to left. For the series-parallel DHT: i-MMD, it has only one EV mode with one EM (1 EM-EV), which means this EV mode needs to cover all the driving situations. Therefore a very large EM2 is necessary for the driving situation with large accelerations or launching in the hill to meet the dynamic requirements. In comparison with the series-parallel DHT, the power-split DHTs including onemode and dual-mode DHT all have the EV mode with 2 EMs. With the aid of EM1, the dynamics in these driving situations can be greatly improved.

The mode distribution of different DHTs in the CS mode is displayed in Fig. 7. To avoid poor engine economic performance in the low-speed range, the series mode in series-parallel DHT and input-power-split mode in powersplit DHT are adopted to improve the fuel consumption. These two modes can uncouple the engine's working points required by wheels to enable better economic performance for the engine. But there is a difference lying between these two kinds of modes as well, namely, the degree and the form of the uncoupling. For series mode, a clutch is used to completely separate the engine power from the wheel, and EM1 as the generator needs to absorb all the engine power to power EM2 or charge the battery. For input-power-split mode, the engine economic performance is also improved through shifting the engine working points to the area with optimal energy consumption with the help of two EMs, but the engine working points are lightly constrained by certain kinematical and kinetic relationships because of the layout. Because of this, there always exists a part of 


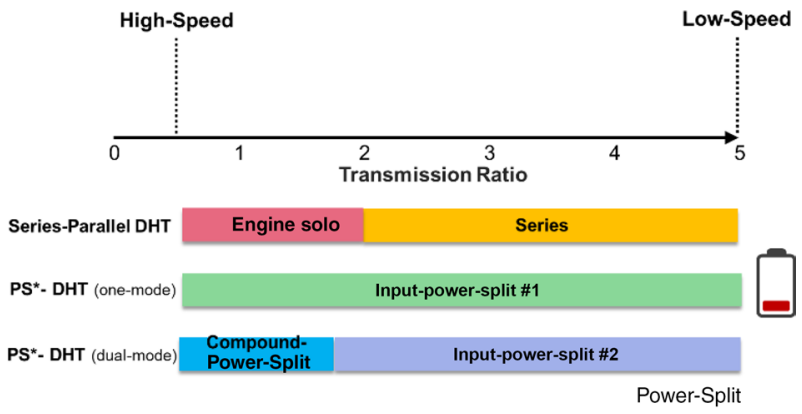

Fig. 7 The comparison of mode distribution in CS mode

engine power flowing through the mechanical path to drive the wheel, which can reduce the power lost in the energy transition as in series mode. Under a relatively higher speed, the direct driving for the engine is efficient. For series-parallel DHT, engine-solo or parallel mode is chosen to drive the vehicle. Regarding input-power-split DHT \#1 for one-mode DHT, most of the engine power, because of the mechanical point, will directly drive the vehicle also in a mechanical way. From this sense, the input-power-split mode is equivalent to the combination of series and parallel mode in the function but has differences in the structure as well. However, the power circulation in input-power-split mode under overly high vehicle speed will limit the reachable maximum vehicle speed. Therefore, for dual-mode DHT, an extra compound-power-split mode is added to solve this problem.

The series-parallel and power-split DHT share some similarities in function, but what are the differences in power requirements for two EMs in different DHTs? Because the DHT with small EM power can reduce the total cost of DHT. In Fig. 8, the $y$-axis is added and represents the absolute value of the power ratio between EM1 and engine over different transmission ratios. For the series-parallel DHT, the power ratio in series mode
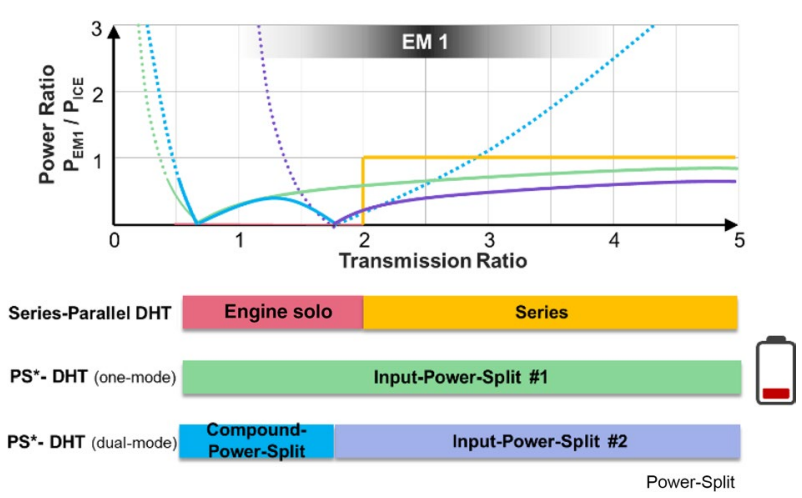

Fig. 8 The comparison of EM power requirement reaches the maximum value of 1, because the EM1 is used as the generator to absorb all the engine power. But under high speed, the engine can drive the vehicle without the aid of EM power. For the input-power-split in one- and dual-mode DHT, the power ratio decreases with the decline of the transmission ratio, and after the mechanical point (the points where the power ratio is 0 for pwersplit DHT), however, it increases again. To compare the EM power requirements for different DHTs, their kinematic equations are derived without considering the inertia; when the battery power doesn't output any net power, the power ratio between EM1 and engine for different power-split modes is shown in Table 3. $i_{\mathrm{g}}$ is the transmission ratio between the engine and transmission output (final drive not included), and $i_{01}$ and $i_{02}$ are the gear ratio of planetary gear sets. The subscripts can be found in Figs. 4 and 5.

The power ratio reaches the maximum value of about 0.85 and 0.69 for the input-power-split \#1 and inputpower-split \#2 at the large transmission ratio, respectively. The dotted line in Fig. 8 means the efficiency of the corresponding modes is too low. The mechanical point of input-power-split \#2 coincides with one of the mechanical points of compound-power-split in dual-mode DHT. It enables the easier mode transition from input-powersplit \#2 to compound-power-split [11]. Theoretically, the corresponding shifting element $\mathrm{C} 1$ can be engaged without the slipping through the mechanical point in the two eCVTs or power-split transitions' process, because at this point, the driving status of the system, including the speed and torque, remains the same. Besides, the EM power requirement of dual-mode DHT is smaller than that of one-mode DHT. The dual-mode DHT effectively reduces the power requirement of EM with the extra hybrid driving mode. From the analysis, the combination of different modes in two kinds of DHTs is necessary and reasonable in function. The success of these DHTs lies in the realization of the necessary functions with the lowest price and reasonable composition.

In a nutshell, the series-parallel DHT is simple in structure but needs large EM to develop the maximum potential of the layout compared with the power-split DHT. The mechanical complexity (namely the complexity of the structure) and electric complexity (namely the requirement of the EM power) always co-exist in one DHT layout. The effective DHT layouts should achieve an excellent balance between mechanical complexity and electrical complexity. The above conclusion is based on the fact that the mechanical complexity and electrical complexity should be effective and not redundant. A concept with many driving modes doesn't necessarily mean it is a good one, because the different driving modes may overlap in the function. Besides, due to the high cost of the electric motors taken in DHTs, the electrical complexity should also be limited to a certain level with the 
Table 3 The derived equations of power ratio for different driving modes

\begin{tabular}{ll}
\hline Mode & Power ratio: $P_{\mathrm{EM} 1} / P_{\mathrm{ICE}}$ \\
\hline Input-power-split \#1 (THS-IV) & $\left(i_{01}+i_{\mathrm{g}}-i_{01} i_{\mathrm{g}}\right) /\left(i_{\mathrm{g}} i_{01}-i_{\mathrm{g}}\right)$ \\
Input-power-split \#2 (Voltec II) & $\left(i_{01} i_{\mathrm{g}}-i_{01}+1\right) /\left(i_{01} i_{\mathrm{g}}\right)$ \\
Compound-power-split (Voltec II) & $\left(i_{01} i_{02} i_{\mathrm{g}}-i_{01} i_{02}+1\right.$ \\
& )$\cdot\left(i_{01} i_{\mathrm{g}}-i_{01}+1\right) /$ \\
& $\left(i_{01} i_{02} i_{\mathrm{g}}-i_{01} i_{\mathrm{g}}\right)$ \\
\hline
\end{tabular}

compensation of mechanical complexity, such as multi-gears or modes like dual-mode DHT.

\section{Dynamics of Series-Parallel DHT and Power-Split DHT}

After the discussion of structure and function for different DHTs, the dynamic comparison is conducted using the reachable driving area of different modes, because the driving area of different modes can, on the one hand, reflect the dynamic performance, and on the other hand demonstrate the mode distribution. In Fig. 9, the driving requirements of all the driving conditions are displayed in the form of wheel torque over different vehicle speeds. The maximum wheel torque reaches about $3000 \mathrm{~N} \cdot \mathrm{m}$ due to the limitation of road adhesion. Theoretically, the driving requirement should be covered by different driving modes.

For the power sources in the analysis, the maximum engine power equals $75 \mathrm{~kW}$; and for the two EM, they are 48 and $87 \mathrm{~kW}$. The comparison of the reachable driving areas of different driving modes is conducted in three driving situations according to the real driving situations:

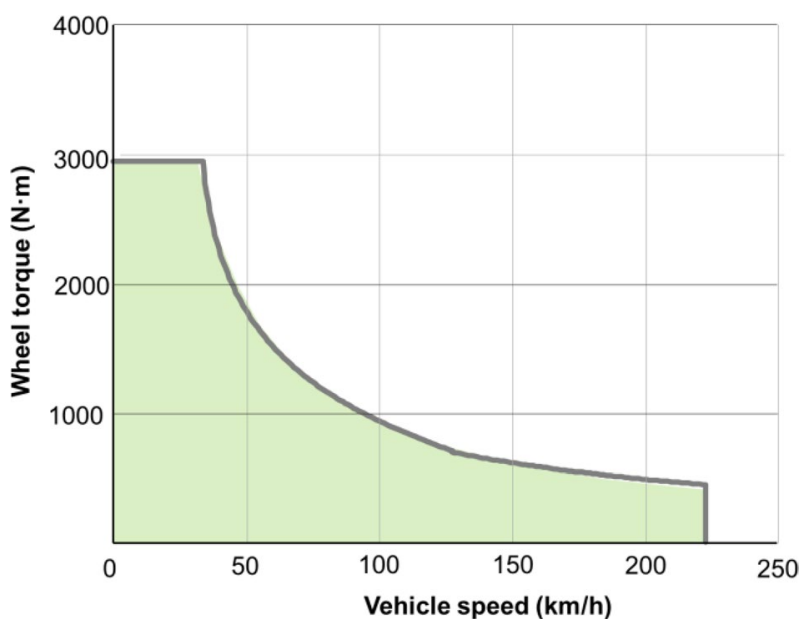

Fig. 9 Driving requirements of all the driving conditions
1. EV-driving (SOC high): only the pure electric modes are used to drive the vehicle.

2. Hybrid-driving (SOC high): the engine and battery power are used to drive the vehicle in hybrid modes under the strict dynamic requirement.

3. Hybrid-driving (SOC low): the output power of the battery is 0 and the electric driving is impossible, but the driving motor can use the power, which is generated from the engine by the generator to drive the vehicle.

\subsection{EV-Driving (SOC High)}

In EV modes in Fig. 10, the series-parallel DHT has only EV-1 with one EM, but power-split DHTs have EV-1 with one EM and EV-2 with two EMs. The driving ranges of EV-1 in two kinds of DHTs are mostly the same. The maximum wheel torque in EV-1 is lower than the wheel torque limit; thus the dynamics of launching in hill or large acceleration cannot be guaranteed by EV-1. But the extra EV-2 mode in power-split DHT can ensure dynamic performance in such driving situations. To sum up, power-split DHTs have a great advantage in dynamics under EV driving mode.

\subsection{Hybrid-Driving (SOC High)}

For hybrid-driving (SOC high) in Fig. 11, the driving range of series mode is the same as that of in EV-1. It means that even with the aid of engine power, the dynamics in low vehicle speed is still relatively poor due to the limitation of EM2 power. With the increase in vehicle speed, the parallel mode can be adopted to accelerate the vehicle and the maximum wheel torque in parallel mode is far beyond the driving requirement. This phenomenon reflects the conflict in the designing of the EM power for series-parallel DHT. To meet the dynamic performance in the launch, a large EM is a necessity, but for middle and high speed, this large EM is excessive. For input-eCVT \#1 in one-mode DHT,

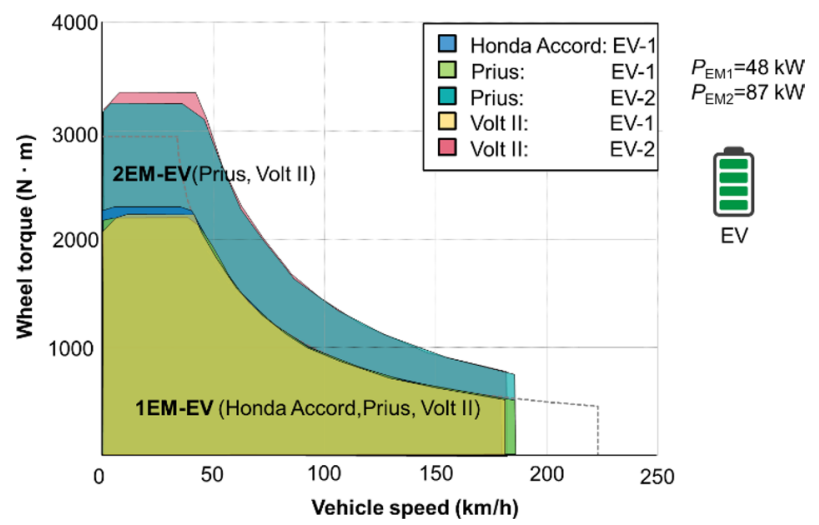

Fig. 10 Driving range of EV mode 


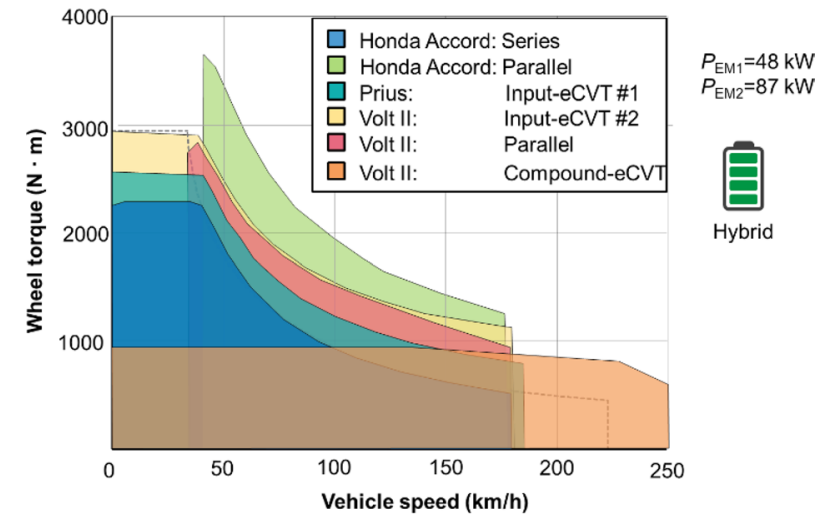

Fig. 11 Driving range of hybrid mode in high-SOC battery

the driving range lies between the series and parallel mode. The maximum torque is larger than that of series mode but smaller than the required maximum wheel torque. For the input-eCVT \#2, its driving range covers the required maximum driving torque exactly and the compound-eCVT mode further extends the maximum vehicle speed without increasing the maximum EM speed. Because in other modes, the maximum vehicle speed all stays around $175 \mathrm{~km} / \mathrm{h}$.

Another interesting phenomenon is that the maximum wheel torque of series and power-split mode are both smaller than that of the parallel mode. The gear ratio in parallel mode has great influence, but the more important reason is the working principle of these modes. For parallel mode, the maximum torque from the engine and EM can be directly or linearly combined to drive the vehicle, but for series and power-split mode, one EM always works as the generator to adjust the engine working point and only the other EM can propose the vehicle in maximum torque. In this sense, the series and power-split modes are more "efficient" in improving engine fuel consumption, and parallel mode is more "dynamic" in driving the vehicle.

\subsection{Hybrid-Driving (SOC Low)}

This driving situation usually appears in low SOC. The dynamic requirement under this situation can be reduced to a certain extent, but the basic driving situations still need to be met. As shown in Fig. 12, the dynamics of series-parallel DHT is relatively weaker, and then comes the one-mode DHT. Only the dual-mode DHT has most of the required torque area covered.

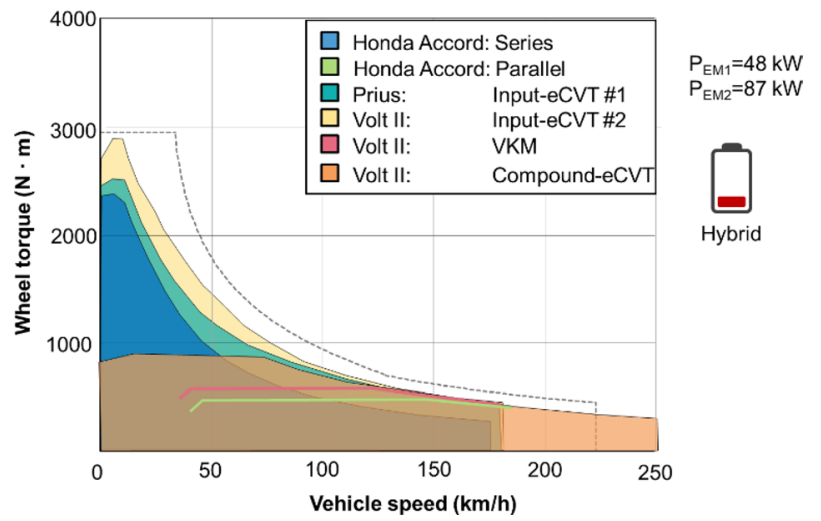

Fig. 12 Driving area of hybrid mode in low-SOC battery

\section{Modular Simulation Model and Optimal Control Strategy}

To evaluate the efficiency and performance of power systems, a modular simulation model for various power system concepts and topologies has been developed in house. Its purpose is to optimize and evaluate different types of powertrains from the transmission and ICE synthesis tool. The simulation model is based on the backward calculation. The vehicle parameters and driving cycles are contained in the module "wheel". According to this information, the driving resistances can be calculated, which can provide the output torque and output speed of the transmission module. In the transmission module, a detailed description of the transmission is included, such as the mechanical connections, defined shift elements, all the transmission operating modes, and shifting logic. Regarding the transmission efficiency, the loss maps are adopted. Then these pre-determined transmission loss maps are integrated into the entire vehicle model. Besides, the transmission module also includes a different number of integrated electric motors that mean both the conventional and the hybrid powertrains, such as DHT, can be predefined and simulated in this module. Further, all possible combinations of operating points are calculated for each driving mode. By this, the required speed and torque in the transmission input can be obtained. Speed and torque from the transmission are delivered to the launch element module. In consideration of the launch element, the output of the launch element module is directly connected to the transmission module. The transmitting element in the launch element may be a disc clutch, a torque converter, or a direct connection. The efficiency behavior of EM and ICE is also modeled using static loss maps. ICE maps are generated 


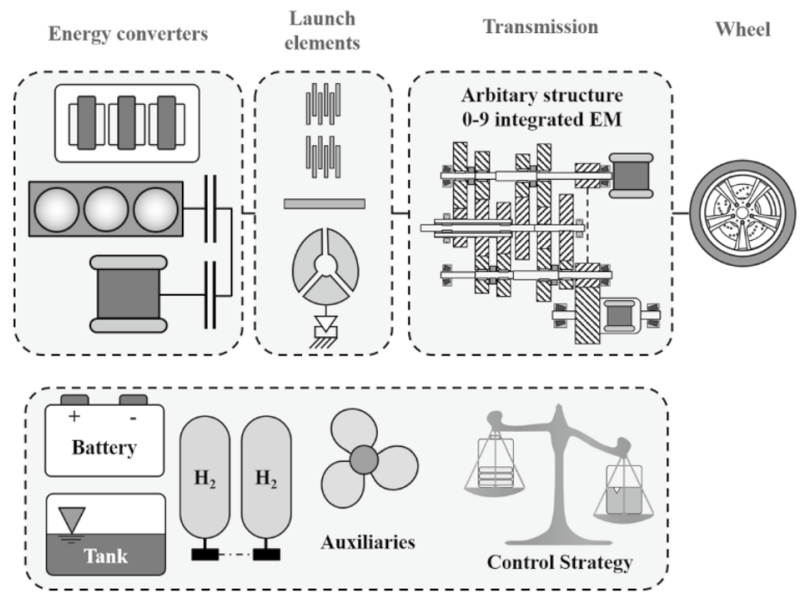

Fig. 13 Modular simulation model

by the ICE synthesis described above. Figure 13 shows the structure of the modular simulation model.

The global optimal control strategy (GOCS) $[12,13]$ is an in-house developed control strategy to determine fuel consumption and energy consumption. The detailed introduction of the GOCS can be found in Ref. [13]. The global energetic optimum is achieved by the systematic sequence of partial optimization. This allows a detailed analysis of the hybrid concepts in consideration of the loss in the process of direct mechanical driving, electric driving, recuperation, and hybrid driving. Besides, the optimization method of this model is specially designed to allow low computational time, which is particularly advantageous for parametric studies with many basic parameter configurations.

\section{Fuel Consumption of Series-Parallel and Power-Split DHT}

The economics of different DHTs is compared in this section. But as mentioned above, even with the same layout, the fuel consumption performance can be largely different with different configurations of parameters and power sources. The optimal configurations are of vital importance to vehicle performance [14]. Therefore, before the comparison of economic performance, the optimization of the configurations should be carried out. The economics of different DHTs can only be compared when all the DHTs are in their optimal configurations. In this way, the influence of configurations on the performance of DHT can be overlooked. In the simulation, a PHEV vehicle is chosen as the target vehicle and its data are shown in Table 4.

The optimization methods like genetic algorithm (GA) and particle swarm optimization (PSO) are usually used in the optimization, but experience shows that these optimizing methods with such a large number of variables are almost
Table 4 The properties of the target PHEV

\begin{tabular}{ll}
\hline Properties & Value \\
\hline $\mathrm{m} / \mathrm{kg}$ & 1500 \\
$\mathrm{c}_{\mathrm{W}} \cdot \mathrm{A} / \mathrm{m}^{2}$ & 0.59 \\
$\lambda$ & 1.03 \\
$f_{\mathrm{R}}$ & $8 \times 10^{-3}$ \\
$P_{\mathrm{ICE}} / \mathrm{kW}$ & 75 \\
$P_{\mathrm{EM} 1} / \mathrm{kW}$ & 48 \\
$P_{\mathrm{EM} 2} / \mathrm{kW}$ & 87 \\
\hline
\end{tabular}

impossible. In this paper, all the combinations of different configurations are simulated. Here the engine power is fixed at $75 \mathrm{~kW}$ as demonstrated in Fig. 14, because the engine is usually determined at the initial developing stage.

The power of two EMs is varied by different maximum rotating speed and torque, as displayed in Fig. 15, and the corresponding efficiency maps are stretched and compressed as well. For the gear ratios and final drive ratio, they are varied in a certain range as well, as displayed in Fig. 16.

For example, for the same one-mode DHT, there are 8125 configurations for the powertrains as displayed in Fig. 17 after the variation of EM power and gear ratios. All the powertrains are further simulated in the modular simulation model described above.

The driving cycle used in Fig. 18 is customer-oriented. It consists of city road, country road, and high speed, which provides a complete and practical cycle in the investigation of fuel consumption.

Another important fact that needs to be considered is that all the different DHTs with varying configurations should meet the minimum dynamic requirements. For example, a configuration with a long acceleration time or low maximum vehicle speed may have better economic performance. In

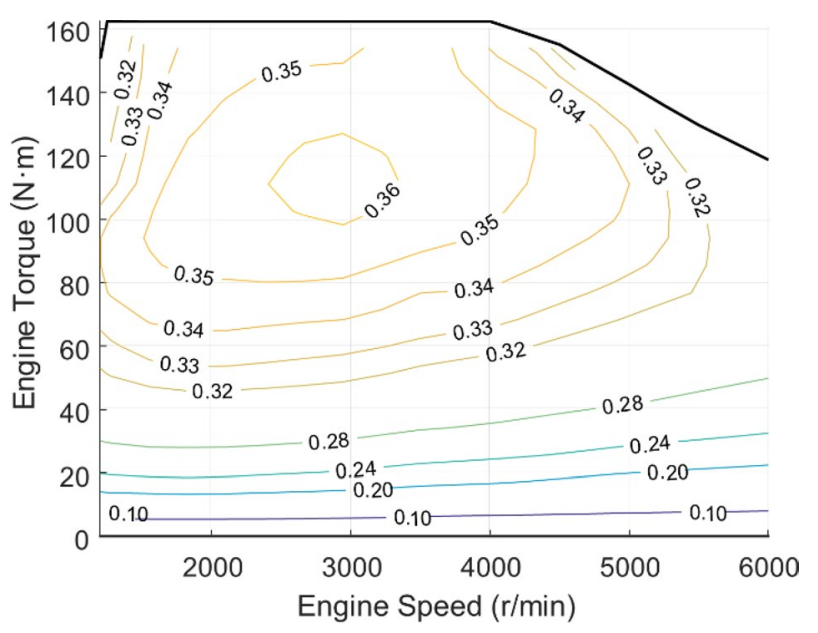

Fig. 14 Engine map \#1 in $75 \mathrm{~kW}$ 


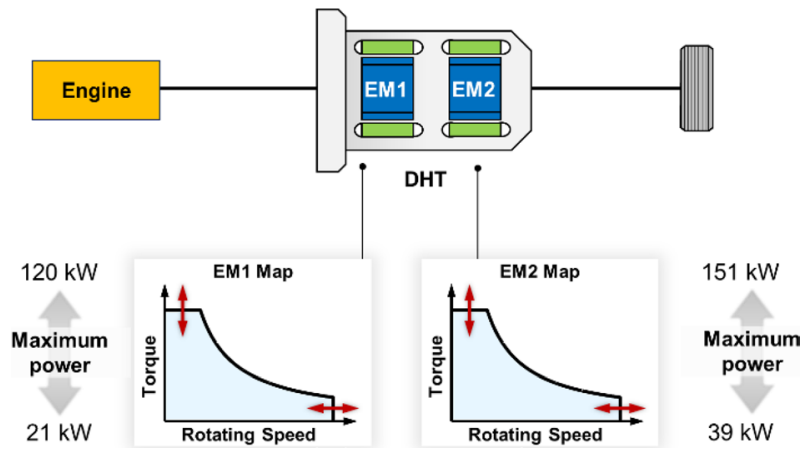

Fig. 15 Variation of EM Power

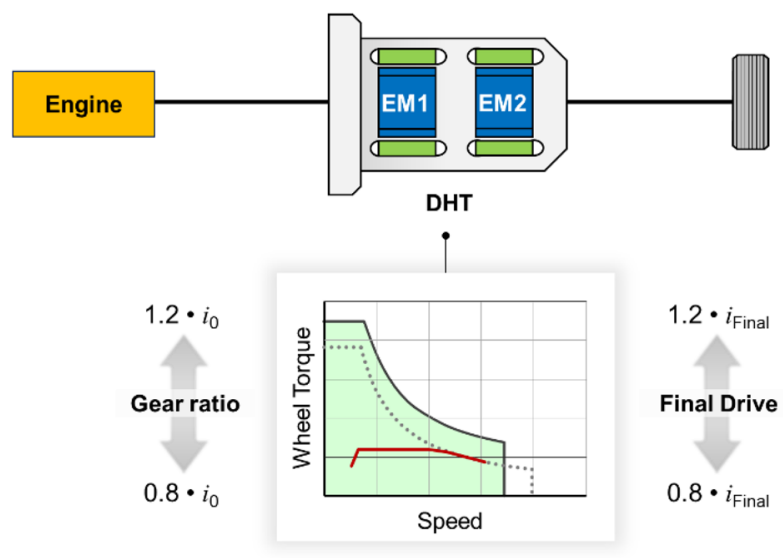

Fig. 16 Variation of gear ratios and final drive

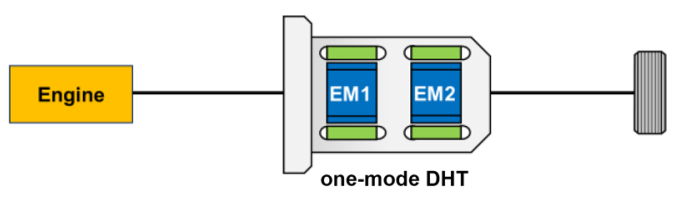

Parameter variation of one-mode DHT:

- Maximum Torque of EM1

- Maximum Torque of EM2

- Gear ratio of PGS1

- Ratio between EM2 and Output

- Final Drive

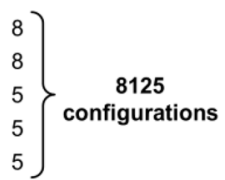

Fig. 17 Different configurations of powertrains for one-mode DHT

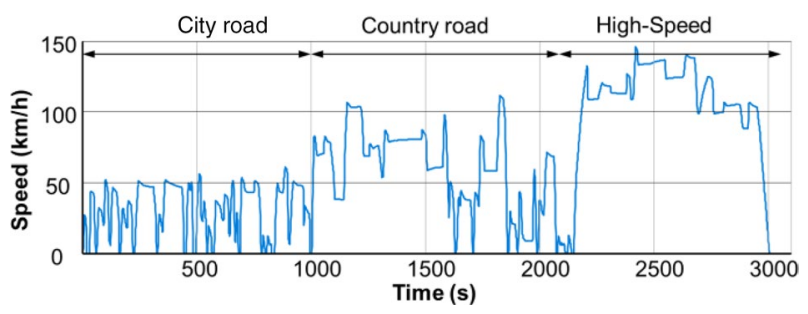

Fig. 18 The driving cycle this sense, the minimum dynamic requirements are defined as follows, and these requirements are also acknowledged by the OEMs in Germany:

1. Maximum vehicle speed in EV and engine-solo mode $\geq 120 \mathrm{~km} / \mathrm{h}$

2. Maximum vehicle speed in hybrid mode (SOC low) $\geq 160 \mathrm{~km} / \mathrm{h}$

3. Gradeability in launch $\geq 30 \%$

4. Acceleration time under $100 \mathrm{~km} / \mathrm{h}$ in EV-driving and hybrid-driving (SOC low) mode $\leq 15 \mathrm{~s}$

After the dynamic simulation, the configurations, with which the minimum dynamic requirements are not met, are filtered. As displayed in Fig. 19, there still exist 4187 configurations for the powertrain.

After the filtering, all the rest configurations of different DHTs are simulated in the driving cycle. To guarantee the effectiveness of comparison, in the control strategies (GOCS) the SOC at the beginning and end of the simulation is controlled to stay at $50 \%$. After the simulation, all

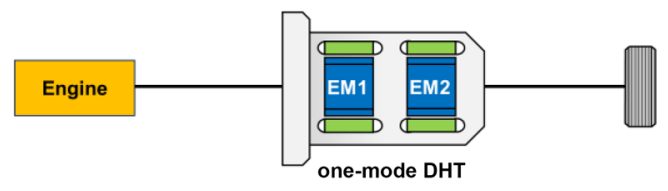

Parameter variation of one-mode DHT:

- Maximum Torque of EM1

- Maximum Torque of EM2

- Gear ratio of PGS1

- Ratio between EM2 and Output

- Final Drive

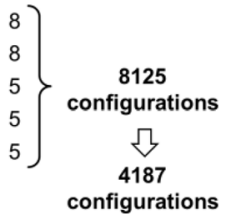

Fig. 19 The filtering of the configurations with minimum dynamic requirements

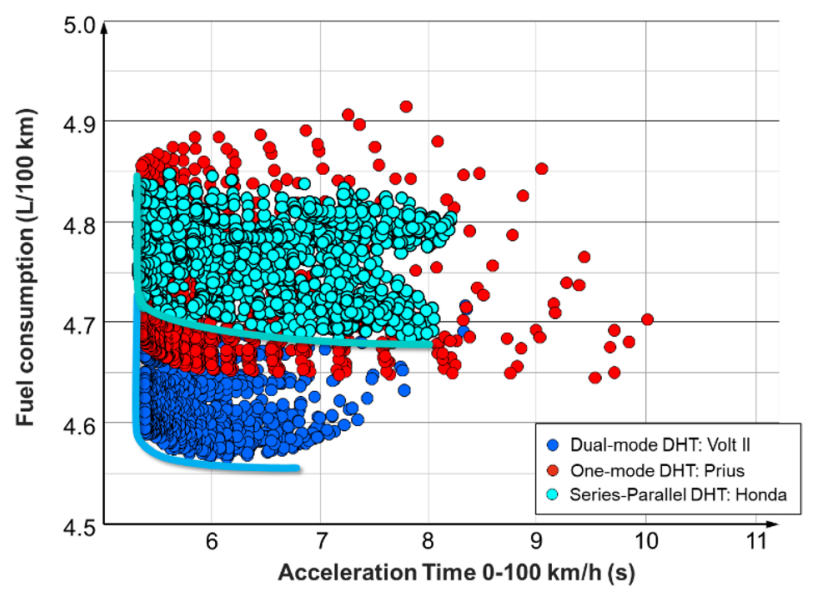

Fig. 20 The simulation results of different configurations for different DHTs 
of the simulation results are plotted in Fig. 20. Every point in the figure represents one configuration of the DHT. The $x$-axis is the acceleration time from 0 to $100 \mathrm{~km} / \mathrm{h}$ in hybrid driving (SOC high). The $y$-axis is the fuel consumption per $100 \mathrm{~km}$ in the driving cycle.

As displayed in Fig. 20, the dual-mode DHT, in general, has a greater advantage in the fuel consumption for different configurations in comparison with other DHTs. The fuel consumption of the one-mode DHT distributes in a relatively large area with different configurations. Different configurations directly determine the performance of the DHT. Regarding the series-parallel DHT, the fuel consumption for most configurations falls in the above area, namely relatively poor economics. Besides, due to the limitation of road adhesion, the shortest acceleration time from 0 to $100 \mathrm{~km} / \mathrm{h}$ for different DHTs stays mostly the same.

In Fig. 21, assuming that the acceleration time from 0 to $100 \mathrm{~km} / \mathrm{h}$ equals $6.5 \mathrm{~s}$ in hybrid driving (SOC high), the optimal consumption of dual-mode DHT, one-mode DHT, and series-parallel DHT are 4.56, 4.65, and 4.70 L, respectively. There exists no obvious large difference in the optimal consumption for different DHTs. That means that with different configurations, different DHTs can obtain the same economic performance. This fact is only valid for the relatively excellent DHT layouts.

In the following, the required EM power for different DHTs to achieve the optimal economic performance is further studied in Fig. 22. The corresponding sum of the EM power for the dual-mode DHT, one-mode DHT, and series-parallel DHT equals 108,136 , and $181 \mathrm{~kW}$, respectively. These results are in agreement with the analysis of the required EM power in the comparison of the function for different DHTs. The series-parallel DHT and power-split DHT all have their pros and cons. The dynamics and economics can both be achieved with the proper configurations. In detail, the series-parallel DHT has a great advantage because of its simple structure but has a disadvantage for the required EM power. Dual-mode DHT is the

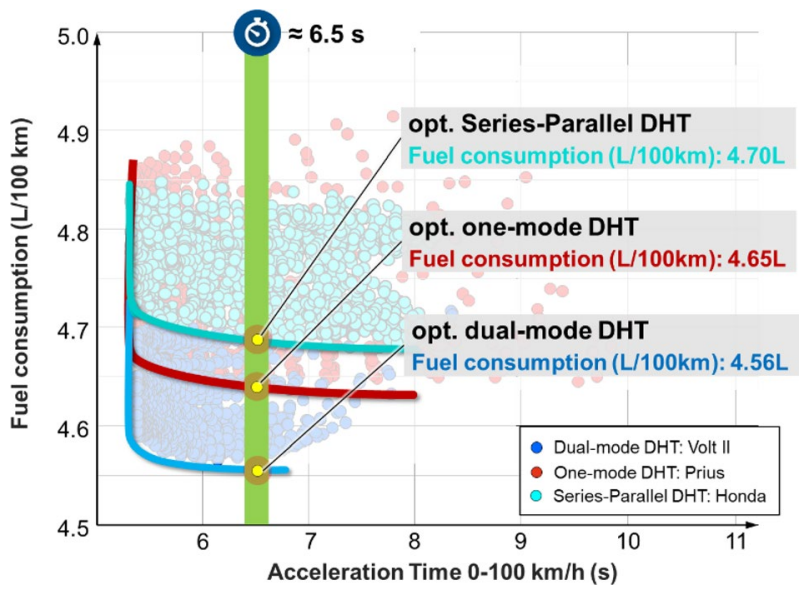

Fig. 21 The comparison of fuel consumption

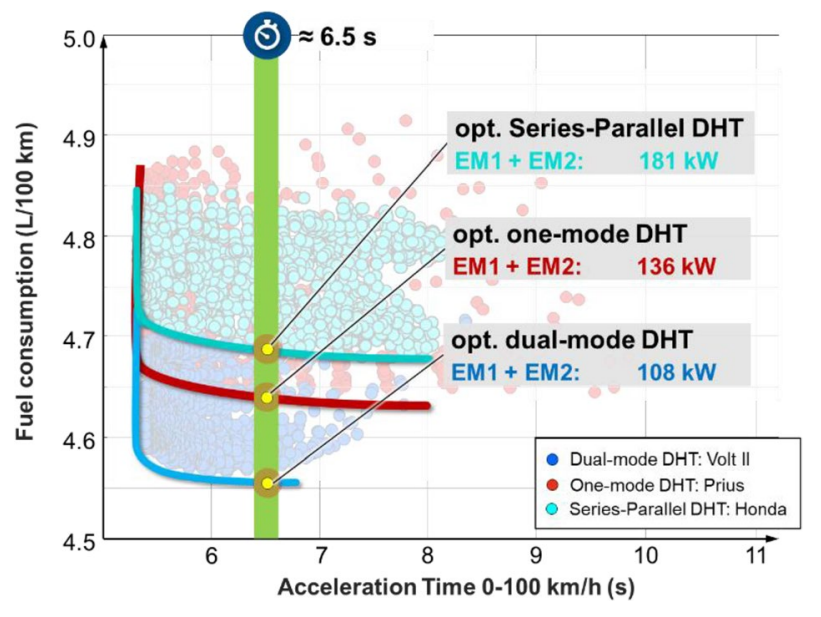

Fig. 22 The comparison of the EM power in the optimal fuel consumption

Table 5 The comparison of fuel consumption with different engine maps

\begin{tabular}{llll}
\hline $\begin{array}{l}\text { Fuel Consump- } \\
\text { tion (L/100 km) }\end{array}$ & $\begin{array}{l}\text { Series-Parallel } \\
\text { DHT: } i \text {-MMD }\end{array}$ & $\begin{array}{l}\text { One-mode } \\
\text { DHT: Prius IV }\end{array}$ & $\begin{array}{l}\text { Dual-mode } \\
\text { DHT: Voltec } \\
\text { II }\end{array}$ \\
\hline Engine map \#1 & 4.70 & 4.65 & 4.56 \\
Engine map \#2 & 4.45 & 4.36 & 4.21 \\
Reduction & $5.32 \%$ & $6.24 \%$ & $7.68 \%$ \\
\hline
\end{tabular}

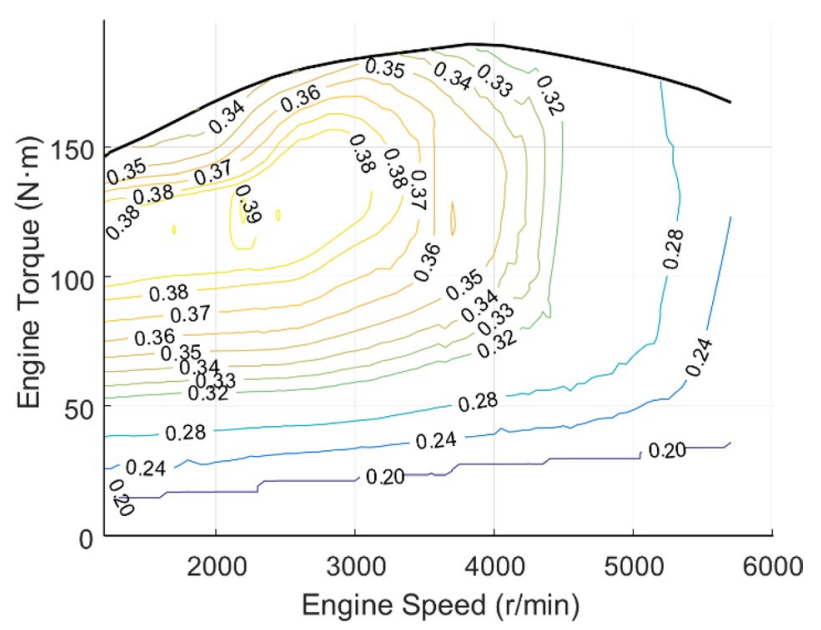

Fig. 23 Engine map \#2 in $75 \mathrm{~kW}$

opposite: it has a low requirement for EM power but a complex structure. The one-mode DHT reaches a compromise between the series-parallel DHT and dual-mode DHT.

In the above analysis, the engine map maintains the same for different DHTs. But what are the influences of different engine maps on different DHTs? For the two kinds of DHTs, 
is the performance of the DHT also sensible to the engine performance? To answer this question, two engine maps with the same power but different characteristics are chosen. The first engine \#1 map is already described in Fig. 14 with the best efficiency of $36.5 \%$ and used in the simulation as well. The second engine \#2 is $1.6 \mathrm{~L}$ with the best efficiency of $40 \%$, which is displayed in Fig. 23. Regarding the economics, engine \#2 performs better than engine \#1.

The simulation for different DHTs with the optimal configurations (namely the optimized results of the gear ratios and EM power) with engine \#2 is further conducted. The results are listed in Table 5. From the simulation results, the fuel consumption with engine map \#2 in $100 \mathrm{~km}$ for series-parallel DHT, one-mode DHT, and dual-mode DHT is $4.45,4.36$, and $4.21 \mathrm{~L}$, respectively. In comparison with the simulation with engine map \#1, dual-mode DHT has $7.68 \%$ lower fuel consumption, and then comes the one-mode DHT with $6.24 \%$. For the series-parallel DHT, the reduction of fuel consumption is $5.32 \%$. These results show that with a better engine map, the fuel consumption of all the DHTs is decreased and power-split DHTs are more sensitive to the engine maps compared with the series-parallel DHTs.

It is seen that the power-split DHTs do have advantages in fuel consumption and need relatively smaller EM. But the structure and control strategies of the power-split DHT are more complex than that of the series-parallel DHT.

\section{Conclusions}

The features and comparisons of different well-known DHTs are discussed and analyzed. The advantages and disadvantages of different kinds of DHT (series-parallel DHT and power-split DHT) in the structure, function, and performance are investigated. Through the comparisons, the main conclusions are drawn as below:

1. Series-parallel DHT and power-split DHT both have advantages and disadvantages in structure and performance. With the proper configurations, the two kinds of DHTs can achieve the same dynamic and economic performance.

2. Series-parallel DHT has low mechanic complexity but high electric complexity (requirement of EM power), while power-split DHT is the opposite. The two kinds of complexities exist in all DHTs. If the DHTs' layouts are effective and not redundant, one complexity can only be reduced with the increase of the other.

3. In comparison with series-parallel DHT, the fuel consumption of power-split DHT is more sensible to the improvement of engine characteristics.
Funding Open Access funding enabled and organized by Projekt DEAL.

Open Access This article is licensed under a Creative Commons Attribution 4.0 International License, which permits use, sharing, adaptation, distribution and reproduction in any medium or format, as long as you give appropriate credit to the original author(s) and the source, provide a link to the Creative Commons licence, and indicate if changes were made. The images or other third party material in this article are included in the article's Creative Commons licence, unless indicated otherwise in a credit line to the material. If material is not included in the article's Creative Commons licence and your intended use is not permitted by statutory regulation or exceeds the permitted use, you will need to obtain permission directly from the copyright holder. To view a copy of this licence, visit http://creativecommons.org/licenses/by/4.0/.

\section{References}

1. Gassmann, T., Hildebrandt, W., Haupt, J., Herber, S.: Efficient multi-mode transmission for plug-in hybrid vehicles. ATZ Worldw. 120(12), 22-27 (2018)

2. Xu, X.Y., Dong, P., Liu, Y., Zhang, H.: Progress in automotive transmission technology. Automot. Innov. 1(3), 187-210 (2018)

3. Ehsani, M., Gao, Y.M., Longo, S., Ebrahimi, K.: Modern Electric, Hybrid Electric, and Fuel Cell Vehicles. CRC Press, Boca Raton (2018)

4. Guo, H., Sun, Q., Wang, C., et al.: A systematic design and optimization method of transmission system and power management for a plug-in hybrid electric vehicle. Energy 148, 1006-1017 (2018)

5. Yang, H., Cho, S., Kim, N., et al.: Analysis of planetary gear hybrid powertrain system part 1: input split system. Int. J. Automot. Technol. 8(6), 771-780 (2007)

6. Schmidt, M. R.: Three-mode, input-split hybrid transmission. US Patent 5,730,676 (1998)

7. Yang, H., Kim, B., Park, Y., et al.: Analysis of planetary gear hybrid powertrain system part 2: output split system. Int. J. Automot. Technol. 10(3), 381-390 (2009)

8. Taniguchi, M., Yashiro, T., Takizawa, K., et al: Development of new hybrid transaxle for compact-class vehicles. SAE Technical Paper 2016-01-1163 (2016)

9. Wang, W., Song, R., Guo, M., et al.: Analysis on compound-split configuration of power-split hybrid electric vehicle. Mech. Mach. Theory 78, 272-288 (2014)

10. De Pinto, S., Mantriota, G.: Power flows in compound transmissions for hybrid vehicles. Machines 7(1), 19 (2019)

11. Conlon, B., Barth, M., Hua, C., et al.: Development of hybridelectric propulsion system for 2016 Chevrolet Malibu. SAE Int. J. Altern. Powertrains 5(2), 259-271 (2016)

12. Chen, H., Li, L., Schudeleit, M., et al.: Composition platform for conventional and hybrid powertrains. SAE Technical Paper 201724-0172 (2017)

13. Lange, A., Küçükay, F.: A new, systematic approach to determine the global energy optimum of a hybrid vehicle. Automot. Engine Technol. 1(1-4), 35-46 (2016)

14. Kim, J., Kim, T., Min, B., Hwang, S., Kim, H.: Mode control strategy for a two-mode hybrid electric vehicle using electrically variable transmission (EVT) and fixed-gear mode. IEEE Trans. Veh. Technol 60, 793-803 (2011) 